\title{
THE PLANETARY MASSES AND THE ORBITS OF THE FIRST FOUR MINOR PLANETS
}

\author{
J. SCHUBART \\ Astronomisches Rechen-Institut, Heidelberg, B.R.D.
}

\author{
(Presented at IAU Colloquium No. 9, 'The IAU System of \\ Astronomical Constants', Heidelberg, Germany, August 12-14, 1970.)
}

\begin{abstract}
Numerical tests are the basis of a study about the effects caused in the orbits of the planets (1)-(4) by possible errors in the system of planetary masses. The masses of five major and three minor planets are considered. Especially, the effects caused by (1) Ceres in the orbit of (2) Pallas since the time of discovery are found to be large enough for a determination of the mass of Ceres. A first result for this mass is $(6.7 \pm 0.4) \times 10^{-10}$ solar masses.
\end{abstract}

\section{Introduction}

The first four minor planets were discovered in the years between 1800 and 1810 . A large number of observations covering more than $160 \mathrm{yr}$ is available for these planets. It is the purpose of this paper to find out, whether the present set of planetary masses is sufficiently accurate to allow a theoretical representation of these observations. Especially, the accuracy in the prediction for the motion of the orbital planes and of the longitudes of the perihelia is considered. It is an important question, whether the masses of the four minor planets can be neglected in a theory of their orbits.

Some of these problems can be solved by computing secular perturbations of the orbital elements of the small planets, but this is not true for all of them. Soon after the discovery of (2) Pallas, Gauss (1874) realized that its mean motion is close to that of (1) Ceres and that, according to this fact, there is a possibility to determine the masses of the two planets from observations accumulated during a long interval of time. Indeed, the synodic period of Ceres with respect to Pallas is about $2000 \mathrm{yr}$, and, according to this, the masses of the two planets will cause long-period effects with a comparatively large amplitude in the mutual mean longitudes. Since the method of secular perturbations is not suitable to study these effects, all the problems mentioned above were treated by means of numerical integration. A number of tests showed the influence of the single masses on the orbits of the four asteroids. Some of the results are given here, together with the result of a first determination of the mass of Ceres from observations of Pallas.

\section{Numerical Tests}

The $n$-body program by Schubart and Stumpff (1966) allows a test of the influence of a planetary mass on the orbits of the four asteroids by the numerical integration of comparatively simple $n$-body cases ( $n=5$ or 6 ). The combined mass of the Sun and Mercury was taken as body No. 1 , while bodies 2-5 represented the first four minor 
planets. To test the action of a major planet, a sixth mass of small amount was put on the orbit of the planet and added to the system. Suitable sets of mean elements were used to derive the starting values of the bodies, since the real perturbing masses were not present in the system. With the exception of the perturbing body considered, the masses of bodies 2-6 were put equal to zero. Table I describes nine computations of this kind, which cover the interval from JD 2380000.5 to 2440000.5 (1804 to 1968) with a steplength of 4 days. In Table $I$, the values of $m_{6}$ are rough guesses of the uncertainties in the conventional values of the respective planetary masses, while estimates of the total mass are used in case of the minor planets. The masses of Ceres and Pallas were derived from the measured diameters and an assumed mean density, which equals that of the Moon. A direct determination of the mass of (4) Vesta by Hertz (1968) is available. A rounded value is used here. The diameter of (3) Juno indicates, that its mass is comparatively small. Therefore, the mass of Juno was not considered in the tests.

TABLE I

\begin{tabular}{|c|c|c|c|}
\hline & $n$ & perturbing mass $^{\mathbf{a}}$ & on orbit of \\
\hline (1) & 5 & - & - \\
\hline (2) & 6 & $m_{\mathbf{6}}=30$ & Earth \\
\hline (3) & 6 & $m_{6}=30$ & Mars \\
\hline (4) & 6 & $m_{6}=300$ & Jupiter \\
\hline (5) & 6 & $m_{6}=3000$ & Saturn \\
\hline (6) & 6 & $m_{6}=3000$ & Uranus \\
\hline (7) & 5 & $m_{2}=$ & (1) Ceres \\
\hline (8) & 5 & $m_{3}=$ & (2) Pallas \\
\hline (9) & 5 & $m_{5}=$ & (4) Vesta \\
\hline
\end{tabular}

Computation No. 1 provided a list of the unperturbed mean longitudes for a number of dates. The other computations were inspected for changes in the orientation of the orbits and for deviations from a linear dependence on time in case of the mean longitudes, since these deviations will appear as observable effects in the observations. Tables II and III contain the results. $\Delta i, \Delta k=\sin i \cdot \Delta \Omega$ and $\Delta \tilde{\omega}$ describe the motion of the orbits. They were obtained from the differences between the osculating elements $i, \Omega$, and $\tilde{\omega}$ at the beginning and at the end of the computations. Therefore, they represent the changes during an interval of $164 \mathrm{yr}$ (in the sense 'end-beginning'). $\Delta l$ shows the largest deviation of the mean longitude, $l$, from a fictitious longitude, which is a linear function of time, and which is defined in each case by the assumption $\Delta l=0$ at the beginning and at the end of the computation. The uncertainties in the motions of the orbital planes and of the longitudes of the perihelia depend mainly on the accuracy of the masses of Jupiter and Saturn. The results for $\Delta i$ and $\Delta k$ indicate a possible total error of 0.1 or 0.2 per century in the motion of some orbital planes. These errors can occur in a theory of the orbits, which is based on a system of planetary masses with errors of the amount given in Table I. Much larger errors occur in the 
TABLE II

\begin{tabular}{|c|c|c|c|c|c|c|c|c|}
\hline \multirow[t]{2}{*}{ Comp. } & \multicolumn{4}{|c|}{ Orbit of Ceres } & \multicolumn{2}{|c|}{ Orbit of Pallas } & \multirow[b]{2}{*}{$\Delta \tilde{\omega}$} & \multirow[b]{2}{*}{$\Delta l$} \\
\hline & $\overline{\Delta i}$ & $\Delta k$ & $\Delta \tilde{\omega}$ & $\Delta l$ & $\Delta i$ & $\Delta k$ & & \\
\hline (2) & $0 " 00$ & $0^{\prime \prime} 00$ & $+0^{\prime \prime 05}$ & 0.0 & $0 " 00$ & $-0^{\prime \prime} 01$ & $+0 \% 01$ & 0 \\
\hline (3) & 0.00 & -0.01 & +0.10 & 0.0 & 0.00 & -0.02 & 0.00 & -0.1 \\
\hline (4) & 0.00 & -0.05 & +0.26 & 0.0 & +0.03 & -0.11 & -0.04 & -0.2 \\
\hline (5) & -0.01 & -0.04 & +0.35 & 0.0 & +0.03 & -0.15 & +0.03 & 0.0 \\
\hline (6) & 0.00 & 0.00 & +0.03 & 0.0 & 0.00 & -0.02 & +0.01 & 0.0 \\
\hline (7) & - & - & - & - & -0.02 & -0.02 & +0.01 & +3.7 \\
\hline (8) & 0.00 & 0.00 & -0.01 & -0.9 & - & - & - & - \\
\hline (9) & 0.00 & 0.00 & +0.03 & -0.4 & 0.00 & 0.00 & 0.00 & 0.0 \\
\hline
\end{tabular}

TABLE III

\begin{tabular}{|c|c|c|c|c|c|c|c|c|}
\hline \multirow[t]{2}{*}{ Comp. } & \multicolumn{4}{|c|}{ Orbit of Juno } & \multicolumn{4}{|c|}{ Orbit of Vesta } \\
\hline & $\Delta i$ & $\Delta k$ & $\Delta \tilde{\omega}$ & $\Delta l$ & $\Delta i$ & $\Delta k$ & $\Delta \tilde{\omega}$ & $\Delta l$ \\
\hline (2) & 0.00 & 0 & $+0: 01$ & $0 " 0$ & 0 & $0 " 00$ & $+0^{\prime \prime} 05$ & $0: 0$ \\
\hline (3) & 0.00 & -0.02 & 0.00 & -0.3 & 0.00 & -0.02 & +0.27 & +0.1 \\
\hline (4) & -0.01 & -0.08 & +0.17 & 0.0 & 0.00 & -0.02 & +0.23 & 0.0 \\
\hline (5) & 0.00 & -0.07 & +0.24 & 0.0 & 0.00 & -0.02 & +0.25 & 0.0 \\
\hline (6) & 0.00 & -0.01 & +0.03 & 0.0 & 0.00 & 0.00 & +0.03 & 0.0 \\
\hline (7) & +0.01 & -0.01 & -0.04 & 0.0 & +0.02 & 0.00 & +0.07 & +1.5 \\
\hline (8) & 0.00 & 0.00 & 0.00 & 0.0 & 0.00 & 0.00 & -0.01 & +0.1 \\
\hline (9) & 0.00 & 0.00 & -0.02 & -0.1 & - & - & - & -- \\
\hline
\end{tabular}

mean longitudes, if the masses of the minor planets are neglected. The values of $\Delta l$ clearly demonstrate the necessity to include the mutual attraction of Ceres, Pallas and Vesta in a theory of their orbits, if all known observations are used. The reason for the interaction of Ceres and Pallas was mentioned before. In case of Ceres and Vesta, the reason is given by a unique, long-lasting approach. The two planets stayed within a distance of 0.3 AU from each other for about 500 days in the years from 1892 to 1894.

\section{The Mass of Ceres}

The values of $\Delta l$ in Table II indicate a chance to determine a reliable value for the mass of Ceres from the observations of Pallas. The effects shown for Mars and Jupiter follow small periods in comparison with those caused by Ceres. To determine a first result for the mass of Ceres, 47 normal positions for the interval from 1803 to 1910 , given by Struve (1911), were combined with 27 positions of Pallas from 1927 to 1968. The computations started with Duncombe's new orbits of Ceres and Pallas (1969). Duncombe's orbit of Pallas represents excellently the modern positions after 1927, but it leaves systematic negative residuals $\cos \delta \cdot \Delta \alpha$ in the old observations. In two oppositions with small geocentric distance, the residuals reach about $\cos \delta \cdot \Delta \alpha=-40^{\prime \prime}$. 
It is possible to represent all the observations of Pallas by a differential correction, if the mass of Ceres is introduced as a seventh unknown, together with the six orbital elements. The method described by Zech (1968) was used. The mass of Ceres results as $m=(6.7 \pm 0.4) \times 10^{-10}$ solar masses, while there are only small corrections to Duncombe's elements of Pallas. Therefore, the estimate for the mass of Ceres in Table I was too low. The same may be true for the mass of Pallas. This should be considered in using Tables II and III. The result for the mass of Ceres (Schubart, 1970) corresponds to the reciprocal value $m^{-1}=(1.5 \pm 0.1) \times 10^{9}$. Mean errors are given.

\section{Acknowledgments}

I thank Professor Dr W. Fricke for directing my attention to the orbits of the first four asteroids and Dr R. L. Duncombe, U.S. Naval Observatory, for making available his new results on these orbits to us in advance. I used the IBM 7094 computer of Deutsches Rechenzentrum in Darmstadt, assisted by a grant from Deutsche Forschungsgemeinschaft, and the Siemens 2002 computer at our institute.

\section{References}

Duncombe, R. L.: 1969, Astron. Papers Am. Ephem. Naut. Alm., Washington, Vol. 20, Part 2.

Gauss, C. F.: 1874, Werke, Band 6, Göttingen, pp. 215-6.

Hertz, H. G.: 1968, Science 160, 299.

Schubart, J.: 1970, IAU Circular, No. 2268.

Schubart, J. and Stumpff, P.: 1966, Veröffentl. Astron. Rechen-Institut Heidelberg, No. 18.

Struve, G.: 1911, Thesis, Berlin.

Zech, G.: 1968, Veröffentl. Astron. Rechen-Institut Heidelberg No. 21. 